\title{
A complement to the theory of biological evolution: individual evolution and genetic mechanism hypothesis
}

\author{
Huichun YUAN ${ }^{1 *}$ (D), Lixin XU ${ }^{1}$, Nianhua WANG ${ }^{1}$
}

\begin{abstract}
In this article, by studying some clinical cases of myopia patients and performing the related analyses, we proposed a conjecture for the causes of myopia and its underlying genetic pathway. Based on that, we designed an experiment to prove the hypothesis of individual evolution. Individual evolution refers to the process of individual trait changes caused by certain external environmental factors, which can be inherited to the next generation. The generation (n) of cloned mice will be classified into X (n1, n1...nx) groups, and the daily exercise amount of each group will be defined as Tx (exercise time). Firstly, we will compare the changes in weight and muscle volume among all groups. Subsequently, the first generation of cloned mice will be used to obtain the second generation of cloned mice, and then grouped in the same way. Then, we will evaluate the effects of exercise on gene expression. For the $\mathrm{n}^{\text {th }}$ generation, the expression levels of individual genes will be determined, and the individual evolution related indexes will be measured. Environmental factors can affect the changes in an individual state, and such alteration can be fed back to the genetic pathways and passed from one generation to the next.
\end{abstract}

Keywords: myopia; genetics; feedback pathway; individual evolution; experimental protocol.

Practical Application: Environmental factors can affect the changes in an individual state, and such alteration can be fed back to the genetic pathways and passed from one generation to the next.

\section{Introduction}

The theory of individual evolution is a complement to the principles of biological evolution and the central dogma of genetics. The bacterial resistance to antibiotics indicates that external environmental factors can alter the expression levels of bacterial related genes, and further to change their phenotypes, which in turn affects the process of population evolution (Malcomson \& Mathers, 2017; Kanak \& Yilmaz, 2020). Throughout the evolutionary history of higher organisms, when individuals adapt to the changes in their external environment, whether their phenotypic traits could lead to genetic alterations and pass it from one generation to the next? Does an individual evolution occur during the evolutionary history of a species?

Hence, the purpose of this conceptual study was to explore the occurrence of individual evolution. Through observing the prevalence of myopia in a selected family, we proposed a conjecture that there are changes in the traits and individuals' genetic predisposition to myopia, and such defectiveness can be passed to the next generation through a genetic pathway (Felberbaum \& Küpker, 2015; Abdullah et al., 2020; Altuntas \& Korukluoglu, 2019; Kanak \& Yilmaz, 2020). In addition, we hypothesized the feedback pathway of individual evolution and its underlying genetic mechanisms. Furthermore, we designed an experiment to prove the hypothesis of individual evolution.

\section{Clinical cases of myopia patients}

There are three sisters in the selected family. The first- and second-born sisters presented myopia before marriage, and their daughters also had contracted myopia during childhood; while the third-born sister and her son were not myopic. Further inquiries into their medical history revealed that if the parents had no myopia before marriage, the latter generation would have no nearsightedness; if one or both parents had myopia, their children would suffer from myopia.

\subsection{The conjecture of the genetic pathway in myopia patients}

Our conjecture: For some patients, myopia is an acquired disease. The myopia-related trait changes would provide feedbacks to the related genes, and these inheritable traits would be passed from parents to their offspring.

Supplementary Note 1: Changes to the related conditions would transmit signals to the genes that underpin the corresponding conditions. In addition, the change in a single gene could alter the entire genome, and the change in condition would be passed to the next generation by altering some genes.

Supplementary Note 2: The adaptive changes of the individuals' traits would be fed back to the relevant genes in the cells, and by means of macroscopic regulation, the abnormal changes in genes might cause genomic instability in multiple organs of the whole body. This phenomenon is termed as individual evolution. 
Supplementary Note 3: The feedback pathway in an individual condition is the genetics basis of individual evolution, and individual evolution is considered as an important element of species evolution.

\subsection{The hypothesis of genetic pathways}

Hypothesis: the "central dogma" of genetics (Yang, 2010) - an RNA-centered closed loop model

Supplementary notes of the hypothesis

The feedback pathway No. 1: the expression of the individual state can transmit signals to RNA through a certain medium, thus regulating the replication or translation of RNA and thereby affecting the synthesis of protein. As a result, the closed feedback loop 1 could be formed.

The feedback pathway No. 2: Following the feedback pathway No. 1, signals can be transmitted to DNA through a certain medium, regulating the replication or transcription of DNA and thereby affecting the synthesis of protein. As a result, the closed feedback loop 2 could be formed.

The feedback pathway No. 3: the expression state of proteins can directly transmit signals to DNA through a certain medium, thus regulating the replication or transcription of DNA and thereby affecting the synthesis of protein. As a result, the closed feedback loop 3 could be formed.

\subsection{The theoretical basis of the experimental design}

Individual evolution refers to the process of individual trait changes caused by certain external environmental factors, which can be inherited to the next generation. The theoretical basis is the feedback mechanism of genetic pathways. Due to the technical limitation of molecular biology, the specific process of genetic pathway may not be confirmed. However, if some experiments can prove the genetic manifestation of phenotype, or in other words, if we can prove that the intervention (cause) and genetic changes (outcomes) are related to an individual state, then individual evolution is speculated to exist.

Therefore, we need to design an experiment to observed the relationship between the intervention and genetic changes, then further to confirm the process of individual evolution.

\subsection{A proposed experiment design to test the hypothesis}

Experiment title: Effects of different amounts of exercise on muscle volume and gene expression in cloned white mice offsprings

Objective: To understand the impact of exercise on muscle volume and gene expression

Methods: The generation ( $\mathrm{n}$ ) of cloned mice will be classified into $\mathrm{X}(\mathrm{n} 1, \mathrm{n} 1 \ldots \mathrm{nx})$ groups, and the daily exercise amount of each group will be defined as Tx (exercise time). Firstly, we will compare the changes in weight and muscle volume among all groups. Subsequently, the first generation of cloned mice will be used to obtain the second generation of cloned mice, and then grouped in the same way. Then, we will evaluate the effects of exercise on gene expression. For the $\mathrm{n}^{\text {th }}$ generation, the expression levels of individual genes will be determined, and the individual evolution related indexes will be measured.

Conclusion: Environmental factors can affect the changes in an individual state, and such alteration can be fed back to the genetic pathways and passed from one generation to the next.

\section{Discussion}

The occurrence of myopia is closely related to both genetic and environmental factors (Zhao \& Yang, 2013). For this common disease, there are some mechanisms of phenomenal causality. In the patients with myopia, genetic changes might occur and transfer to their offspring; thus, the probability of developing myopia is greatly increased.

We can conjecture that for some patients, myopia is an acquired disease. Their corresponding traits changed after the disease developed, and such changes are inheritable. Alteration in the state of the individual can be transmitted to the statedetermining genes through a medium that changes the state information and spreads it to a variety of cells, including germ cells. Such genetic transmission may require an explanation from updated theories. Here, we proposed a hypothesis based on the central dogma of genetics, in which the transmission of genetic information can occur through an RNA-centered closed loop (Figures 1-4).

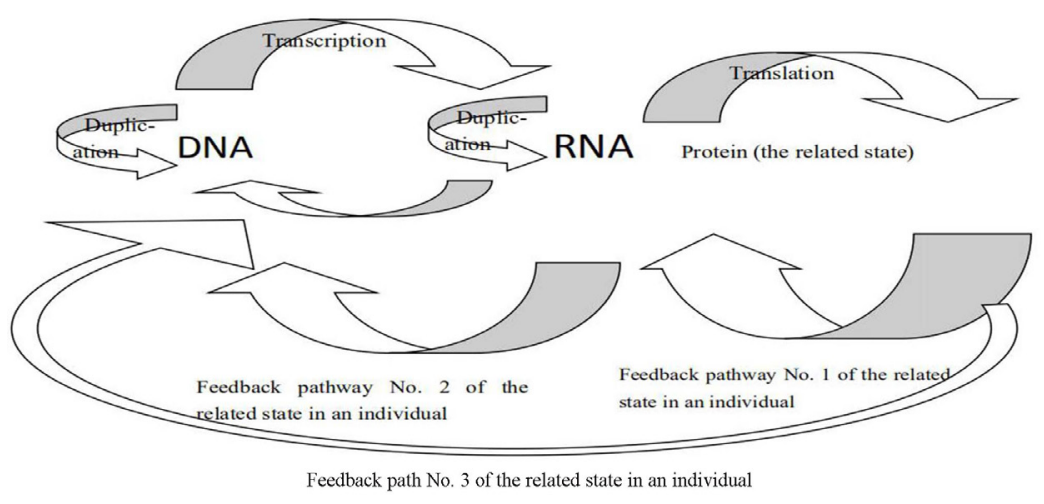

Figure 1. The "central dogma" of genetics. 


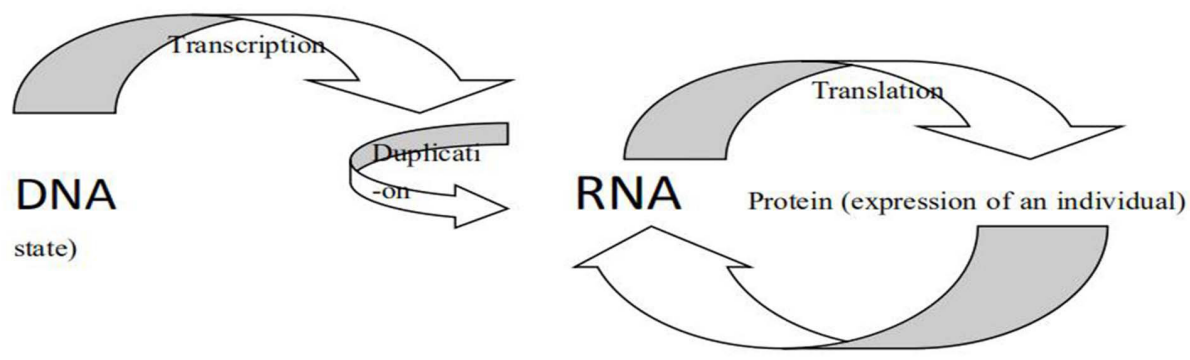

Feedback pathway No. 1 of the related state in an individual

Figure 2. Feedback path No. 1 of the related state in an individual.

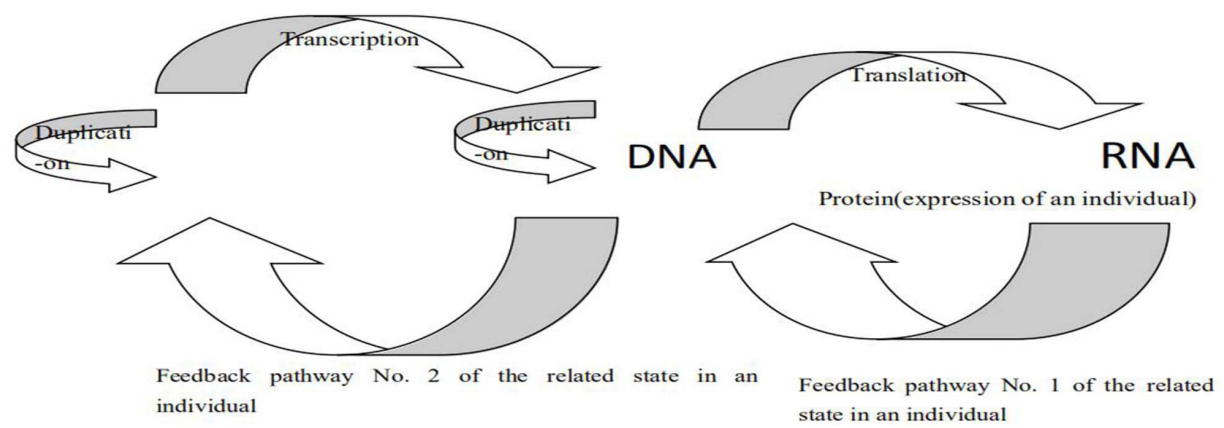

Figure 3. Feedback pathway No. 2 of the related state in an individual.

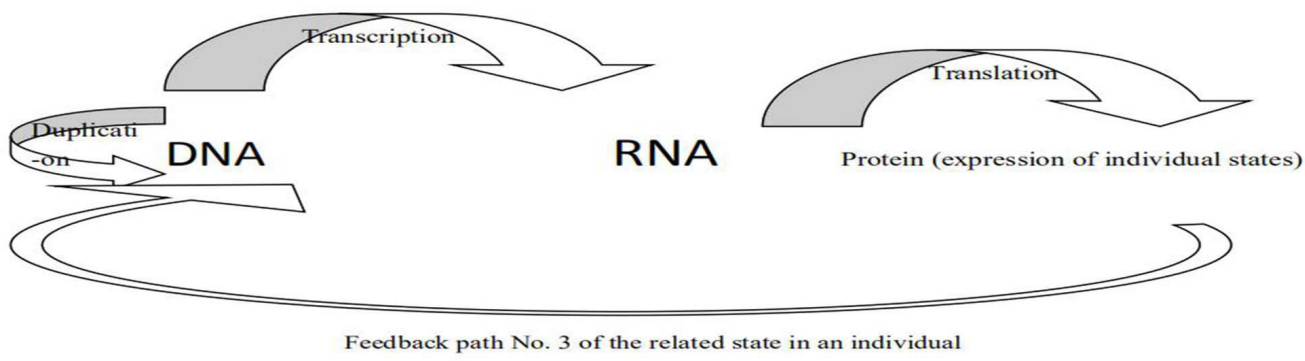

Figure 4. Feedback pathway No. 3 of the related state in an individual.

Nevertheless, our conjectures and hypotheses still need to be proven by experimental evidence, and such experiment has not been performed by any laboratory in the world. The purpose of this conceptual paper is to invite experts and scholars from all over the world to explore this mysterious scientific field.

\section{Acknowledgements}

We appreciate this journal for giving us the opportunity to publish this paper. We also appreciate all those who care about us and help us grow.

\section{References}

Abdullah, M., Mahmood, S., \& Ahmed, Z. (2020). Evaluation of antianemic prospective of natural iron sources in lactating women: an ignored important segment of Pakistani population. Food Science and Technology. In press. http://dx.doi.org/10.1590/fst.05020.
Altuntas, S., \& Korukluoglu, M. (2019). Growth and effect of garlic (Allium sativum) on selected beneficial bacteria. Food Science and Technology, 39(4), 897-904. http://dx.doi.org/10.1590/fst.10618.

Felberbaum, R., \& Küpker, W. (2015). It's all in the genes: is it all in the genes? Gynakologische Endokrinologie, 13(2), 81. http://dx.doi. org/10.1007/s10304-015-0006-6.

Kanak, E. K., \& Yilmaz, S. O. (2020). Identification, antibacterial and antifungal effects, antibiotic resistance of some lactic acid bacteria. Food Science and Technology. In press. http://dx.doi.org/10.1590/ fst.07120.

Malcomson, F. C., \& Mathers, J. C. (2017). Nutrition, epigenetics and health through life. Nutrition Bulletin, 42(3), 254-265. http://dx.doi. org/10.1111/nbu.12281.

Yang, W. (2010). Cell biology (2nd ed., Vol. 8, pp. 225-226). Beijing: People's Medical Publishing House.

Zhao, K., \& Yang, P. (2013) Ophthalmology (8th ed., pp. 246-247). Beijing: People's Medical Publishing House. 